\title{
Accelerated partial breast radiotherapy: a review of the literature and future directions
}

\author{
Nathan R. Bennion ${ }^{1}$, Michael Baine ${ }^{1}$, Andrew Granatowicz ${ }^{2}$, Andrew O. Wahl ${ }^{1}$ \\ ${ }^{1}$ Department of Radiation Oncology, Fred \& Pamela Buffet Cancer Center at the University of Nebraska Medical Center, Omaha, Nebraska, USA; \\ ${ }^{2}$ Nebraska Medicine, Omaha, Nebraska, USA \\ Contributions: (I) Conception and design: All authors; (II) Administrative support: NR Bennion, AO Wahl; (III) Provision of study materials or \\ patients: NR Bennion, M Baine, A Granatowicz, AO Wahl; (IV) Collection and assembly of data: All authors; (V) Data analysis and interpretation: \\ All authors; (VI) Manuscript writing: All authors; (VII) Final approval of manuscript: All authors. \\ Correspondence to: Nathan R. Bennion. Department of Radiation Oncology, Fred \& Pamela Buffet Cancer Center at the University of Nebraska \\ Medical Center, Omaha, Nebraska, USA. Email: nathan.bennion@unmc.edu.
}

\begin{abstract}
Breast conservation therapy exemplifies the tailoring of medicine in the care of patients with cancer. Akin to improvements in surgical approaches, accelerated partial breast irradiation (APBI) tailors the treatment volume and duration to the needs of well selected patients. Here, we examine the evidence supporting APBI as well as the lessons in patient selection, dose and delivery techniques. Examination of historical techniques and their associated outcomes will support more correct patient selection and treatment delivery in an era where we await the reports of several large prospective trials.
\end{abstract}

Keywords: Breast cancer; accelerated partial breast irradiation (APBI); breast conservation; radiotherapy; brachytherapy; toxicity

Submitted Sep 20, 2018. Accepted for publication Nov 12, 2018.

doi: $10.21037 /$ gs.2018.11.05

View this article at: http://dx.doi.org/10.21037/gs.2018.11.05

\section{Introduction}

Breast conservation therapy has ushered in a myriad of patient-tailored treatments. In radiotherapy, it is further tailored by adjusting the therapeutic time, dose, fractionation, and in the case of accelerated partial breast irradiation (APBI), the target volume as well. For decades, adjuvant whole breast irradiation (WBI) allowed for breast conservation with oncologic outcomes comparable to mastectomy without the associated morbidity (1). Adjuvant WBI has shown to reduce the local-regional recurrence by approximately two thirds (1-3) and has even shown a survival benefit (4). Albeit a sound option, investigations appropriately searched for more convenient treatments with lower side effect profiles.

As a reference, conventional WBI targets the entire ipsilateral breast using a pair of opposed beams oriented tangentially to the chest wall (Figure 1). Examples of common dose and fractionation schemes may include hypofractionated (short course) regimens with 15 treatments all the way to conventional fractionation with 25 daily fractions of 2 Gy. Depending on patient and tumor characteristics, an additional 10-16 Gy may also be delivered to the lumpectomy cavity. Pioneers of APBI postulated that longer treatment courses and larger treatment volumes could be more costly, inconvenient, and result in increased toxicity. In response, APBI was conceived as a way to decrease the volume treated and shorten the duration of treatment. It is typically performed either as brachytherapy with a radioactive source delivering treatment from within the breast via treatment catheters or with multiple external beams that converge on the lumpectomy cavity. Treatments typically target the lumpectomy cavity with a predetermined margin and can be delivered in as little as a single week. Intraoperative treatments have also been investigated using electrons or kilovoltage machines but will not be covered in this review.

Arguments to alter approaches to adjuvant WBI after 

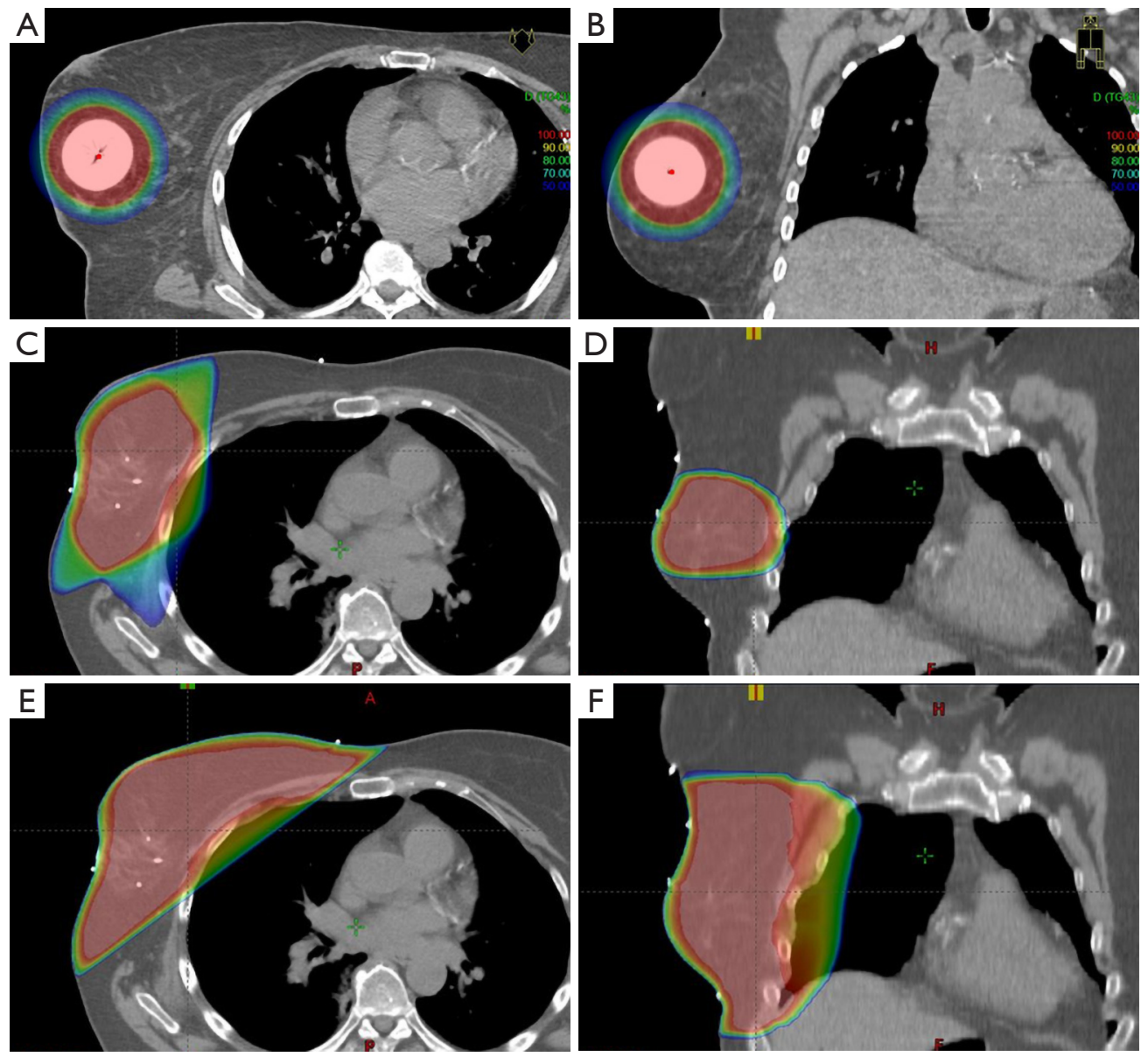

Figure 1 Dose distribution in breast radiotherapy. Axial and coronal dose distributions characteristic of balloon-based brachytherapy (A,B), external beam partial breast irradiation $(\mathrm{C}, \mathrm{D})$, and whole breast irradiation (E,F) respectively.

breast conservation surgery seek to decrease costs, improve convenience for the patient, and attempt to lower acute and long-term side effects. In a modern prospective study, grade 2 or greater acute toxicity for WBI included fatigue $(18 \%)$, radiation dermatitis $(40 \%)$, and pain (4\%) (5). Though long-term side effects occur with lower incidence than their acute counterparts, they play a central role in decision making. Darby et al. estimated that for every mean 1 Gray (Gy) received by the heart, cardiac events rose by a relative $7 \%$ (6). Lymphedema is also a consideration when numbering toxicities. Despite the fact that lymphedema rates are low when treating the breast alone (7), the risk rises as a function of volume and location irradiated $(5,8)$. Even rare side effects such as secondary malignancies have been sufficiently associated with the dose and volume of tissue irradiated (9). In addition to decreasing the target volume, APBI generally offers a shorter, more convenient treatment course. APBI has also been proposed as a more cost-effective approach when compared to WBI (10). Since the 1990's, investigators proposed that APBI could address the negative concerns regarding WBI by decreasing the volume irradiated, the time patients spend under treatment, the acute and long-term side effects experienced, and the financial burden on the patient and community as a whole, while simultaneously improving cosmetic outcomes and quality of life. However, this new treatment has not emerged without scrutiny.

Concerns with the APBI included inadequate oncologic outcomes, technical feasibility, new toxicity profiles, and patient selection. Some questioned whether or not target volume reduction from WBI would result in higher localregional failures or if new toxicities would arise like higher incidences of fat necrosis, skin toxicity or cosmetic outcomes. Last of all, what were the appropriate doses, 
volumes, immobilization to ensure adequate coverage and reproducibility? These questions helped form the basis of early APBI treatment and studies.

\section{Rationale for volumes treated in APBI}

In 1985, Holland et al. published a series of mastectomy specimens with 282 invasive cancers and 32 ductal carcinoma in-situ (11). These were selected as cases that may be appropriate for breast conservation therapy, i.e., no clinical/radiological multifocality, or AJCC stage T3 or T4 tumors. Of the 282 invasive specimens, 37\% showed no tumor beyond the reference mass, $20 \%$ showed additional disease within $2 \mathrm{~cm}$, and $43 \%$ demonstrated disease beyond the $2 \mathrm{~cm}$ radius. Some argue that this earlier cohort presented with more advanced disease and that mammography has changed the pathologic landscape. Prospective trials helped to clarify patterns of failure in the era of breast conservation $(3,12)$. In these studies, tumor bed recurrences predominated in-breast failures with less than 5\% occurring elsewhere. In 2004, Vicini et al. reviewed the pathology from patients who underwent re-excision after lumpectomy prior to radiotherapy. Of those with a negative surgical margin (no tumor on ink) $>90 \%$ of the specimen showed no disease beyond $1 \mathrm{~cm}$ from the initial lumpectomy bed (13). These findings emboldened the APBI argument.

\section{What dose and fractionation are appropriate for APBI?}

Conventional fractionation schemes (1.8-2 Gy per fraction) have been well established both at the bench and bedside to exploit the differences in DNA repair between normal and malignant tissues. Simply put, each tissue has a different radiosensitivity characterized by two coefficients $\alpha$ and $\beta$. When used as a ratio, they describe the magnitude of damage caused by changes in dose per fractionation. For example, tissues with lower $\alpha / \beta$ ratios demonstrate more DNA damage with increases in dose per fraction when compared to tissues with a higher $\alpha / \beta$ ratios. Though the $\alpha / \beta$ ratio for breast cancer was previously thought to be approximately 10, in two European sister prospective studies (START A and START B), it was determined to be $4.1(14,15)$. This $\alpha / \beta$ ratio is similar to the $\alpha / \beta$ ratio of late responding tissue. Lower $\alpha / \beta$ ratios in tumors, like that seen in breast cancer, enables radiation oncologists to exploit the damage caused by higher doses per fraction without unnecessarily increasing the damage to normal tissues. These European studies did in fact demonstrate similar local control rates between the high-dose and low-dose per fraction treatment arms while superior cosmetic outcomes were noted in the high dose per fraction arm.

Using $\alpha / \beta$ ratios, a linear quadratic model is able to estimate responses based on individual tissue characteristics. APBI investigators similarly referenced conventional dose/ fractionation schemes for the breast-typically 50 Gy in 25 fractions-and calculated radiobiologic equivalent doses with higher doses per fraction using the linear quadratic model. Commonly accepted regimens may include $34 \mathrm{~Gy}$ in 10 fractions for brachytherapy or $38 \mathrm{~Gy}$ in 10 fractions with external beam radiotherapy (EBRT).

\section{What is the approach for patient selection?}

Prior to surgery, patient selection relies heavily on a proper workup. Important patient characteristics include reliability for future follow-up, whether or not they are a surgical candidate, other comorbidities and patient anatomy. Clearly, when considering minimal treatment, reliability for future mammograms and physical exams plays a primary role. Patient anatomy must also be considered. For example, breast size relative to tumor bed location would play role in whether or not APBI is feasible, especially in brachytherapy techniques. Tumor characteristics of most importance are those that affect the patient's recurrence risk locally and regionally, such as size, margin status, histology, grade, positive lymph nodes, centricity/focality, lymphovascular space invasion (LVSI) and high-risk genetic mutations. These clinical risk factors have been considered in trial eligibility, as well as consensus guidelines as discussed below. Factors that may affect the technical feasibility of safely delivering APBI may include tumor size, lumpectomy cavity geometry, and distance from the lumpectomy bed to the skin surface.

\section{Which modality should be used?}

With multiple experiences published in each of the modalities, there are several viable techniques using brachytherapy and linear accelerators. Here we will focus on post-surgical techniques. Multicatheter/interstitial and balloon-based brachytherapy each have their strengths and weaknesses, as does external beam APBI. We address these in detail below using selected experiences and data to demonstrate their utility. 


\section{Brachytherapy-based APBI}

The most mature and robust APBI data currently available is in the setting of brachytherapy. While generally considered a singular treatment modality, brachytherapy for APBI can be performed using multiple techniques, each with its own set of supporting data. For the purposes of this review, APBI brachytherapy will be divided into interstitial and applicator-based treatments.

Of the two forms of APBI brachytherapy, interstitial brachytherapy is most dependent on the operator's skill and experience. Placement of the interstitial applicators can be performed either at the time of surgery or at a later time following healing of the surgical scar. Advantages of placement at the time of lumpectomy revolve primarily around the avoidance of a second procedure for catheter implantation, reducing the patient's overall treatment time, and the ability to precisely delineate the location of the lumpectomy bed. However, perioperative catheter insertion risks the potential of needing to abort the procedure should final pathology and margin status make the patient a poor candidate for APBI. In the instance of postoperative catheter insertion following return of final pathology, catheters were placed with the aid of tumor bed visualization through $\mathrm{CT}, \mathrm{X}$-ray and/or ultrasound. The implant quality is dependent on a good understanding of the tumor bed location, often aided by the presence of surgical clips left by the surgeon at the time of lumpectomy (16). These techniques have a relatively long history of use with consistently encouraging outcomes. Specifically, a randomized trial from the Hungarian National Institute of Oncology randomized 258 women with early stage (T1N0$1 \mathrm{mi})$, low grade (grade 1-2), non-lobular breast cancer who underwent lumpectomy with negative margins to receive either WBI without lumpectomy bed boost or APBI via interstitial brachytherapy using a 7 -fraction technique. Currently, 10-year outcome results are available revealing comparable rates of local recurrence $5.9 \%$ with APBI $v s$. $5.1 \%$ with $\mathrm{WBI}$ ) and improved cosmetic outcomes with the partial breast technique (17). These outcomes are further bolstered by the results of the more recent GEC-ESTRO non-inferiority trial which randomized 1,184 patients with stage 0-IIA breast cancer who underwent lumpectomy with negative margins to WBI with inclusion of a tumor bed boost or APBI with interstitial brachytherapy (using either 7- or 8-fraction techniques, delivered twice daily). Fiveyear results revealed equivalent rates of local recurrence ( $1.4 \%$ vs. $0.9 \%$ for APBI and whole breast radiation, respectively). Late grade $2-3$ late skin toxicity was $3.2 \%$ for APBI and $5.7 \%$ for WBI and there was a trend for improved breast pain, favoring APBI $(18,19)$. Importantly, the results of these randomized trials are also very similar to results from previously conducted single arm studies in which multiple interstitial brachytherapy techniques were investigated. Throughout these studies, ipsilateral breast tumor recurrence (IBTR) rates ranged from $5.2-15 \%$ at greater than 10-year follow-up in each study with generally favorable toxicity profiles and cosmetic outcomes (20-24).

Given the limitations in generalizability of the interstitial APBI technique, the feasibility and safety of applicator based APBI brachytherapy evolved as described in 2007 with studies reporting oncologic outcomes starting thereafter (25). In applicator based APBI, the applicator is inserted into the lumpectomy bed and expanded to fill the cavity. Applicators are comprised of a balloon expanded with saline or a series of deformable catheters without a balloon and, like interstitial catheters, can be placed either at the time of surgery or following initial healing and return of final pathology. Regardless of applicator type, each contains between 1 and 8 channels for introduction of the brachytherapy source to provide treatment to the tumor bed with a margin of $10-15 \mathrm{~mm}$ (16). Currently, the largest data in support of this treatment option comes from the American Society of Breast Surgeons (ASBS) Registry which enrolled 1,444 patients comprising 1,449 cases treated with 10 twice-daily fractions of high-dose-rate brachytherapy using a single lumen applicator. In this data set, the 5-year rate of IBTR was 3.8\% with low associated rates of toxicity including $2.5 \%$ rate of fat necrosis, $13.4 \%$ rate of symptomatic seromas, and $9.6 \%$ risk of infection. Of note, subgroup analysis from these patients suggest that lack of estrogen receptor positivity as well as tumor size were associated with increased risks of IBTR (26-28). More recent data from the Contura registry trial which involved 342 patients showed similar results with a $2.2 \%$ risk of local recurrence at 3 years and an even more favorable toxicity profile with rates of symptomatic seroma and infection of $4.4 \%$ and $8.5 \%$, respectively (29). Multiple small, single institution studies have also reported similar results for both local recurrence and toxicity, though are collectively with short follow-up periods of 2 years or less. Importantly, while no prospective randomized trial comparing applicator based brachytherapy to WBI currently exists, a matched pair analysis comparing outcomes for patients enrolled into the ASBS (APBI applicator-based brachytherapy) and Surveillance Epidemiology End Results (WBI) registries 
has been performed indicating no difference in locoregional recurrence between the two modalities (30).

\section{External beam-based APBI}

External beam-based techniques utilize linear accelerators to target and treat partial breast volumes. These techniques may also vary in their beam arrangement, patient position, target localization, dose and fractionation. Early pioneers of this non-invasive technique reported their outcomes from William Beaumont Hospital (31). They defined the clinical target volume (CTV) as the lumpectomy cavity in the supine position plus an additional $10-15 \mathrm{~mm}$ and an additional $1 \mathrm{~cm}$ expansion to planning target volume (PTV), less $5 \mathrm{~mm}$ from the skin surface. There were no IBTR and cosmetic outcomes were encouraging with $90 \%$ of patients scored with a good to excellent cosmesis at 3 or more years from treatment. Alongside was a team at New York University (NYU) that reported their early experiences $(32,33)$. Formenti et al. also demonstrated good to excellent cosmetic outcomes in $100 \%$ of patients initially and no grade 2 or 3 events. The target volume for NYU was slightly different. Patients were treated prone, and the CTV was the lumpectomy bed without any expansions, but PTV used larger expansions of $15-20 \mathrm{~mm}$ from the CTV. With a similar target definition, but in the supine position, Massachusetts General Hospital demonstrated goodexcellent cosmesis in $100 \%$ at 12 months from treatment. Though they did note significant acute skin toxicity.

These results gave rise to more phase II studies (Table 1) (56-59). In 2005, the Radiation Therapy Oncology Group (RTOG) published their initial efficacy results from 0319. Fifty-two evaluable patients again demonstrated $6 \%$ local failure rate and only two grade 3 toxicities. That same year however, the University of Michigan reported unacceptable cosmesis in their cohort of 34 patients treated with 3.85 Gy twice daily fractions to 38.5 Gy with intensity modulated radiation therapy (IMRT) (48). The study was closed due to new development of unacceptable cosmesis in seven patients. Though only one plan deviated from dosimetric requirements, the investigators detected a significant difference in the volumes receiving $50 \%$ and $100 \%$ of the prescription dose between those with acceptable and unacceptable cosmetic outcomes. Later, they reported final cosmetic outcomes with $43.3 \%$ excellent and $30 \%$ good cosmetic scores. There were no grade 3 toxicities however (49). The authors postulated that the poor cosmetic outcome was likely due to larger volumes receiving $20 \%, 50 \%, 80 \%$ and $100 \%$ of the prescription dose despite meeting criteria for the NSABP-B39 protocol. They also noted that their use of breathing control may have decreased inherent feathering of the dose compared to other study's free-breathing technique. A phase III trial, the Canadian RAPID study, randomly assigned women after breast-conservation surgery to WBI (conventional or hypofractionated doses) to APBI with 38.5 Gy in 10 twice daily fractions. The authors reported adverse cosmetic scores between APBI and WBI from nurses (29\% vs. 17\%), patients ( $26 \%$ vs. $18 \%$ ), and physicians ( $35 \%$ vs. $17 \%)$, each favoring cosmesis in the WBI arm. The authors postulated that the dose used had a higher predicted normal tissue biologic effect than commonly used prescriptions for WBI but expected that the smaller irradiated volumes would be compensatory. Aside from dose, they also submitted that the 6-hour interval between fractions may not have been adequate for normal tissue repair.

In the face of emerging data with unexpected poor cosmetic results, other single arm studies continued to produce encouraging results. A multi-institutional Canadian study reported by Berrang et al. used similar dose, fractionation, and volumes as the RAPID study with $86 \%$ of patients achieving good-excellent cosmetic scores (46). Akin to the IMRT used in the Michigan study, the APBIMRT study reported by Lei et al. demonstrated over $90 \%$ goodexcellent cosmesis in 136 patients with a median follow-up of 53 months (44). Again, the Massachusetts led study last reported by Pashtan et al. showed $97 \%$ of patients treated with 3.2 Gy in 8 twice daily had at acceptable cosmetic outcomes with a median follow-up of 71 months (50). These studies showed IBTR rates of $1 \%$ or less.

Several randomized trials compared WBI to external beam APBI (Table 1), albeit with slight variations in the dose and fractionation. A group of investigators from Barcelona randomized 102 patients to APBI [three-dimensional conformal radiotherapy (3D-CRT)] with 3.75 Gy times 10 twice daily fractions versus WBI showing good to excellent patient reported cosmetic outcomes reaching $100 \%$ in the APBI arm (34). Although physicians scored the cosmetic results lower at over $75 \%$ with good to excellent results at a median of 60-month follow-up, patients positive self-scoring and lack of local recurrences gave encouragement for the previously questioned modality, especially when statistics showed no difference between the arms. Multiple Italian reports demonstrated good outcomes with external beam APBI. Meduri et al. reported the IRMA study in abstract form with 3-year 


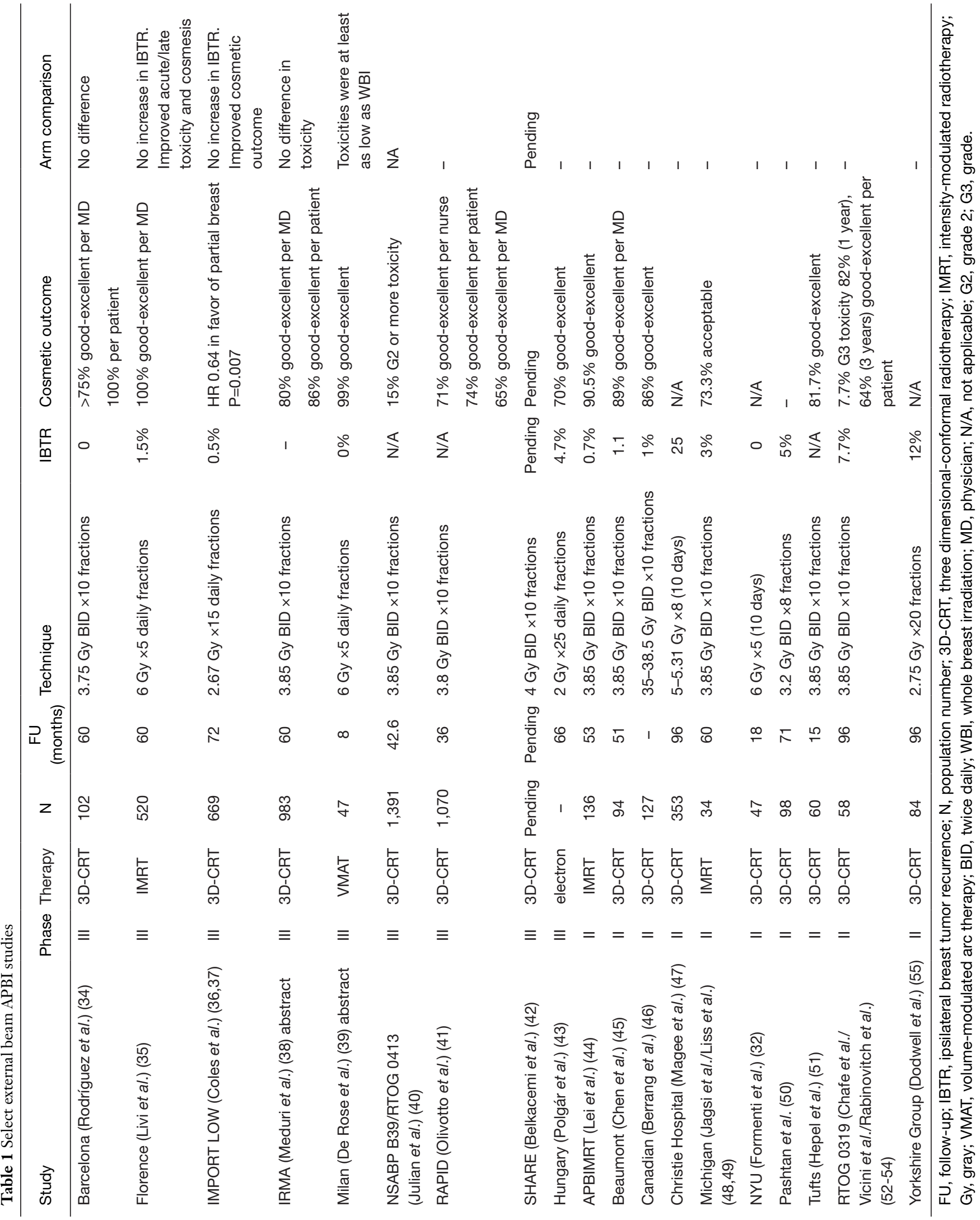


physician and patient reports of unacceptable cosmesis at rates of $20 \%$ and $14 \%$ respectively with 60 -month median follow-up (38). Again, there was no statistical difference in toxicity. Another abstract presented 1 year later at the European Society for Radiotherapy and Oncology by De Rose et al. demonstrated 47 patients treated with volume-modulated arc therapy (VMAT) with 99\% good-excellent cosmesis and no IBTR (39). Both of these abstracts reported at least equivalent toxicity/ cosmesis when compared to WBI. A group of investigators from Florence randomized patients to external beam APBI (IMRT) with 6 Gy $\times 5$ daily fractions versus WBI with 50 Gy in 25 fractions followed by a boost and demonstrated $100 \%$ of the 260 patients enrolled in the APBI arm had goodexcellent cosmesis at 60-month median follow-up (60). There was a $1.5 \%$ IBTR rate in the APBI arm, similar to that found in the WBI arm. In terms of cosmetic outcomes, the APBI arm statistically outperformed those with WBI. The IMPORT LOW trial also showed improved cosmesis in the partial breast cohort albeit in a more protracted course than most APBI protocols (36). This United Kingdom based multicenter trial randomized women with unifocal tumors less than $3 \mathrm{~cm}$ with up to 3 positive axillary nodes to receive 40 Gy WBI (control group), 36 Gy WBI (dose-reduced group) and 40 Gy partial breast radiotherapy in 15 fractions. Partial breast was delivered by modifying the superior and inferior boarders of conventional tangents. Median follow-up reached 72.2 months where 5 -year local relapse was $1.1 \%, 0.2 \%$, and $0.5 \%$ in the control, dosereduced, and partial breast groups respectively. Partial breast irradiation demonstrated lower rates of changes in breast appearance and firmness when compared to the control group. Lastly, the awaited NSABP B39/RTOG 0413 trial published early results in their EBRT APBI arm that showed low rates of grade 2 or greater toxicity of $15 \%$ (38).

In terms of oncologic outcomes, the discussed studies show that appropriately selected patients have acceptable IBTR rates with APBI comparable to those of WBI. Late toxicity and cosmetic outcomes however have been under some scrutiny. Attention to late effects requires diligent follow-up as multiple studies have shown changes in cosmesis between the 1-, 3-, and 5-year follow-up intervals. But the question remains-how do we reconcile the disparities in outcomes from reports of APBI having deleterious effects on cosmesis versus those that report improvements over the current standard of care of WBI? Peterson et al published the predictors of adverse cosmetic outcomes in the RAPID trial (61). Importantly, they noted that clinicopathologic characteristics were similar between APBI and WBI cohorts. In the APBI cohort specifically, they discovered that tumor location, smoking, age, and seroma volumes were statistically significant in their association with adverse cosmesis $(\mathrm{P}<0.05)$. Smoking was also associated with cosmetic deterioration $(\mathrm{P}=0.02)$. They previously hypothesized association of unacceptable cosmesis with the size of the irradiated volume. With more long-term data, they analyzed the ratio of volume receiving at least $95 \%$ of the prescription dose to the whole breast target volume. When using the ratio threshold of 0.15 , they reported a statistically significant association with a $\mathrm{P}$ value of 0.04 . While others have reported correlation of toxicity/cosmesis with radiated volumes, others have found no correlation at all $(51,52)$. Beaumont also conducted a study to determine (62) the predictors of long-term toxicity. Multiple toxicities, including the worst and last incidence of poor-fair cosmesis, were not associated with the absolute breast volume receiving $5-50 \%$ of the prescription dose. Other predictors of toxicity were location and cavity-toskin distance. Even though data of dosimetric correlations with toxicity are conflicting, it is prudent to improve conformality with accurate target delineation, optimal treatment planning and appropriate use of image guided radiotherapy (IGRT) to avoid excessive PTV margins. One issue that remains unaddressed that may account for differences in cosmetic outcomes could be that of surgery's effect on cosmetic outcomes in the setting of APBI.

\section{What are the current guidelines for APBI?}

Based on the current data, the American Society of Radiation Oncology (ASTRO) has set forth a series of guidelines to guide implementation of APBI $(63,64)$. First published in 2009, and later updated in 2017, these guidelines separate patients into three suitability groups based on multiple criteria for the (Table 2). All patients who do not fall into either the suitable or unsuitable categories are considered cautionary and require extensive cost/benefit analysis and discussion with her treating physician to best determine if APBI is a reasonable consideration. Importantly, as discussed in the ASTRO consensus statement update, most data underlying these recommendations are considered weak. Thus, as results from the previously discussed trials mature and data from the currently accruing trials, discussed and subsequent sections, become available it is possible that these recommendations will change. 
Table 2 Current ASTRO guidelines for accelerated partial breast irradiation $(63,64)$

\begin{tabular}{|c|c|c|c|}
\hline Consideration & Suitable & Cautionary & Unsuitable \\
\hline Genetics & BRCA $1 / 2$ negative & - & BRCA 1/2 positive \\
\hline Margins & $\geq 2 \mathrm{~mm}$ & $<2 \mathrm{~mm}$ & Positive \\
\hline Size & $\leq 2.0 \mathrm{~cm}$ & $2.0-3.0 \mathrm{~cm}$ & $>3.0 \mathrm{~cm}$ \\
\hline LVSI & None & Limited/focal & Extensive \\
\hline Focality & Clinically unifocal & - & $\begin{array}{l}\text { Clinically multifocal or } \\
\text { microscopically multifocal with } \\
\text { total size }>3.0 \mathrm{~cm}\end{array}$ \\
\hline Centricity & Unicentric & - & Multicentric \\
\hline Nodal status & $\mathrm{pNO}$ & - & $\mathrm{pN}+$ \\
\hline Neoadjuvant therapy & None & - & Any \\
\hline
\end{tabular}

DCIS, ductal carcinoma in situ; LVSI, lymphovascular space invasion; pNO, pathologically node-negative; ER, estrogen receptor; pN+, pathologically node-positive.

\section{Future directions}

With the heterogeneity of currently published data combined with continued enthusiasm for the potential of APBI, a myriad of trials are currently enrolling patients or awaiting data maturation prior to the reporting of results. At the time of this publication, a total of 34 trials are actively recruiting or closed but yet to be published (Table 3).

Six of the currently active trials are seeking to compare outcomes of APBI to standard of care whole breast radiation therapy following lumpectomy; consisting of five phase 3 trials and a single phase 2 trial $(41,65-67,72,80)$. Importantly, only one phase 3 trial and the phase 2 trial are actively accruing patients with results from the RAPID and NSABP B39 trials imminent. The majority of these trials compare APBI to standard fraction WBI while one phase 3 trial (SHARE) as well as the phase 2 Italian study either allow or require patients enrolled on their respective whole breast radiation arms to be treated with a hypofractionated technique. It is also noteworthy that, while two of the phase 3 trials are comparing conventional fractionation WBI to brachytherapy-based APBI using either balloon-based or interstitial techniques and the sole phase 2 trial is utilizing an external beam APBI regimen consisting of 3,000 cGy over 5 fractions delivered every other day, all other current trials comparing APBI with standard of care techniques utilize external beam partial breast irradiation with a uniform dose and fractionation schedule of 3,850 cGy delivered over 10 twice daily fractions.

Beyond these comparative studies, multiple other active trials are seeking to further improve both our understanding, and delivery, of APBI. A phase 2, nonrandomized Hungarian study is currently seeking to compare 3D-CRT and IMRT techniques in the delivery of external beam APBI, and closed to accrual in 2014 (68). A randomized phase 2 study from Ontario is attempting to delineate the optimal external beam APBI dose, comparing 2,750 to $3,000 \mathrm{cGy}$, both delivered over 5 daily fractions using a $3 \mathrm{D}$-CRT approach in a phase 2 study which is also currently closed to accrual and awaiting results (69). Mayo Clinic recently met accrual for a phase 2 study 


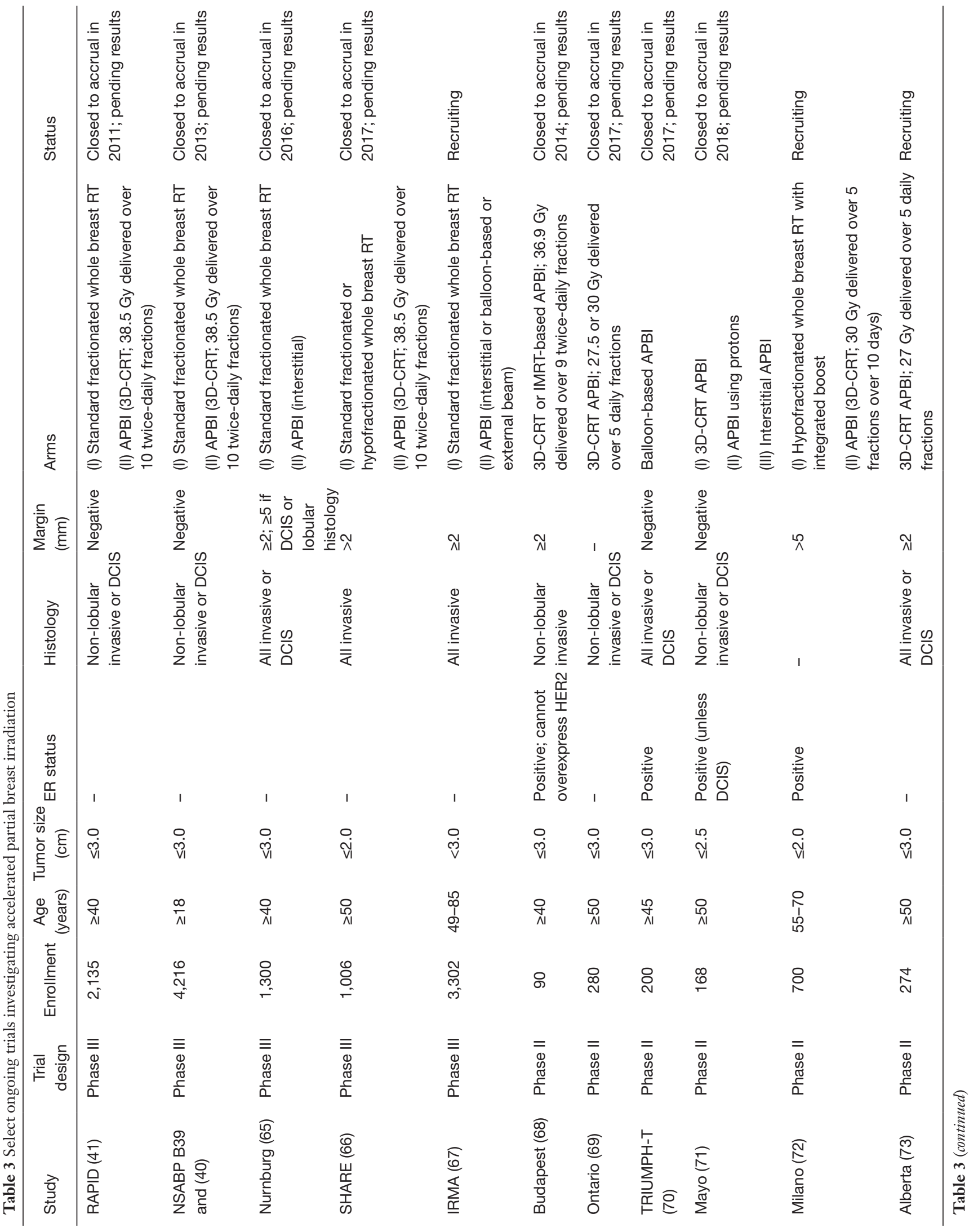




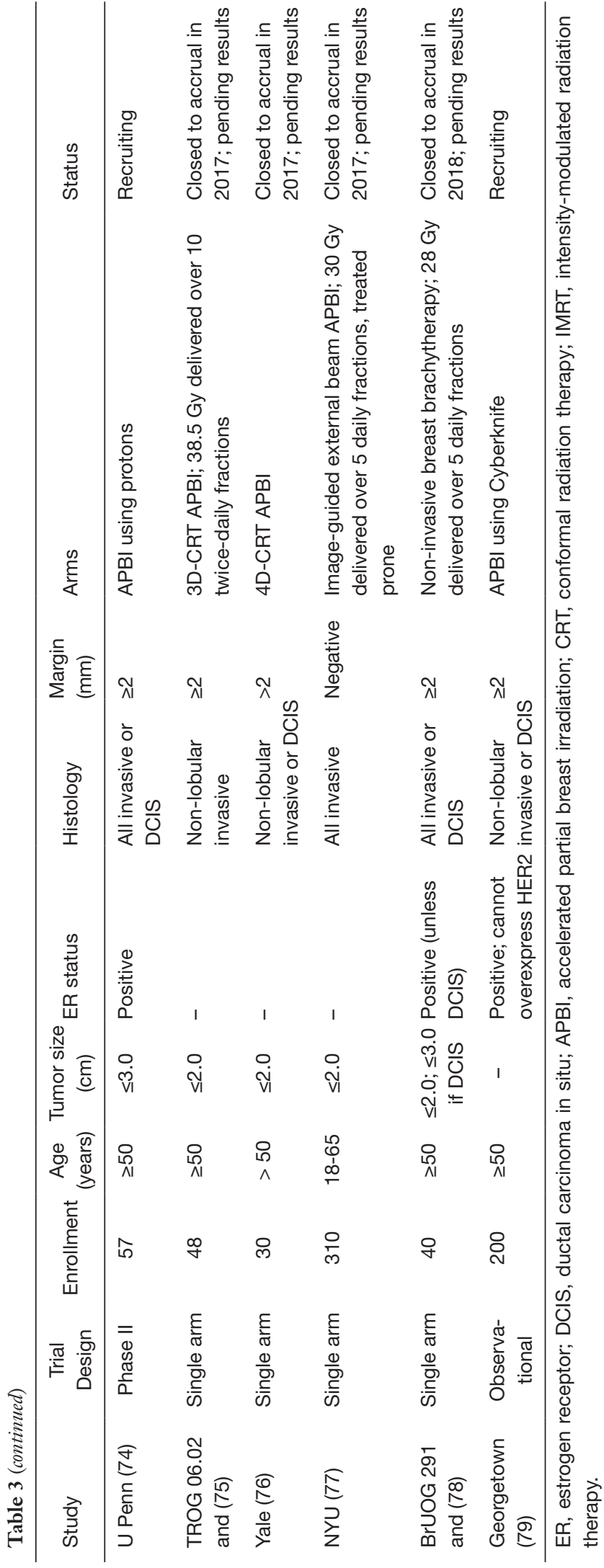

which allowed patient's to be treated with either external beam, protons, or interstitial brachytherapy (71). While not designed to be a definitive head-to-head comparison of these techniques, this study may help to elucidate optimal treatment technique in the setting of partial breast irradiation. Importantly, multiple other institutions, including university of Pennsylvania and MD Anderson, are actively recruiting patients in feasibility studies investigating proton based APBI $(74,81)$. Still other groups are attempting to decrease dose to normal tissue using various positional or modality-based techniques including prone treatment or utilization of breast compression with a noninvasive brachytherapy boost $(77,78)$.

Of note, there are also currently multiple ongoing trials investigating APBI delivered in a neoadjuvant manner which are beyond the scope with his current publication but may represent another avenue of APBI utilization in the future (82-84).

In light of the 5 -year results from previously published works such as GEC-ESTRO and IMPORT LOW as well as the other large cooperative group trials currently recruiting or closed but awaiting results, it is apparent that our understanding of the role of APBI in the treatment of women with early stage breast cancer will expand greatly in the coming years. The heterogeneity of enrollment criteria across the currently active studies will also help to shed light on the relative importance of the various criteria currently utilized in APBI guidelines such as margin status, nodal involvement, histology, hormone receptor status, and patient age. As this data matures, we will undoubtedly be able to better assess patients for their suitability for APBI and separate them from patients who should instead get treatment to the entire breast or may not need any adjuvant radiation therapy at all.

As data matures, more robust information will soon guide physicians and the patients that they care for. Though APBI has certainly moved beyond the realm of experimental treatments, the details of patient selection and treatment techniques will certainly benefit from the level 1 data yet to be reported. Until then, it is prudent to heed the lessons learned from experiences past.

\section{Acknowledgements}

None.

\section{Footnote}

Conflicts of Interest: The authors have no conflicts of interest 
to declare.

\section{References}

1. Fisher B, Anderson S, Bryant J, et al. Twenty-year followup of a randomized trial comparing total mastectomy, lumpectomy, and lumpectomy plus irradiation for the treatment of invasive breast cancer. N Engl J Med 2002;347:1233-41.

2. Liljegren G, Holmberg L, Bergh J, et al. 10-Year results after sector resection with or without postoperative radiotherapy for stage I breast cancer: a randomized trial. J Clin Oncol 1999;17:2326-33.

3. Veronesi U, Marubini E, Mariani L, et al. Radiotherapy after breast-conserving surgery in small breast carcinoma: long-term results of a randomized trial. Ann Oncol 2001;12:997-1003.

4. Early Breast Cancer Trialists' Collaborative Group (EBCTCG), Darby S, McGale P, et al. Effect of radiotherapy after breast-conserving surgery on 10year recurrence and 15-year breast cancer death: metaanalysis of individual patient data for 10,801 women in 17 randomised trials. Lancet 2011;378:1707-16.

5. Whelan TJ, Olivotto IA, Parulekar WR, et al. Regional nodal irradiation in early-stage breast cancer. $\mathrm{N}$ Engl J Med 2015;373:307-16.

6. Darby SC, Ewertz M, McGale P, et al. Risk of ischemic heart disease in women after radiotherapy for breast cancer. N Engl J Med 2013;368:987-98.

7. Akhtari M, Teh BS. Accelerated partial breast irradiation: advances and controversies. Chin J Cancer 2016;35:31.

8. Poortmans PM, Collette S, Kirkove C, et al. Internal mammary and medial supraclavicular irradiation in breast cancer. N Engl J Med 2015;373:317-27.

9. Dörr W, Herrmann T. Cancer induction by radiotherapy: dose dependence and spatial relationship to irradiated volume. J Radiol Prot 2002;22:A117-21.

10. Sher DJ, Wittenberg E, Suh WW, et al. Partial-breast irradiation versus whole-breast irradiation for early-stage breast cancer: a cost-effectiveness analysis. Int J Radiat Oncol Biol Phys 2009;74:440-6.

11. Holland R, Veling SH, Mravunac M, et al. Histologic multifocality of tis, T1-2 breast carcinomas implications for clinical trials of breast-conserving surgery. Cancer 1985;56:979-90.

12. Liljegren G, Holmberg L, Adami H, et al. UppsalaOreboro Breast Cancer Study Group: sector resection with or without post-operative radiotherapy for stage I breast cancer: five-year results of a randomized trial. J Natl Cancer Inst 1994;86:717-22.

13. Vicini FA, Kestin LL, Goldstein NS. Defining the clinical target volume for patients with early-stage breast cancer treated with lumpectomy and accelerated partial breast irradiation: a pathologic analysis. Int J Radiat Oncol Biol Phys 2004;60:722-30.

14. START Trialists' Group, Bentzen SM, Agrawal RK, et al. The UK Standardisation of Breast Radiotherapy (START) Trial A of radiotherapy hypofractionation for treatment of early breast cancer: a randomised trial. Lancet Oncol 2008;9:331-41.

15. START Trialists' Group, Bentzen SM, Agrawal RK, et al. The UK Standardisation of Breast Radiotherapy (START) Trial B of radiotherapy hypofractionation for treatment of early breast cancer: a randomised trial. Lancet 2008;371:1098-107.

16. Skowronek J, Wawrzyniak-Hojczyk M, Ambrochowicz $\mathrm{K}$. Brachytherapy in accelerated partial breast irradiation (APBI) - review of treatment methods. J Contemp Brachytherapy 2012;4:152-64.

17. Polgár C, Fodor J, Major T, et al. Breast-conserving therapy with partial or whole breast irradiation: ten-year results of the Budapest randomized trial. Radiother Oncol 2013;108:197-202.

18. Strnad V, Ott OJ, Hildebrandt G, et al. 5-Year Results of Accelerated Partial Breast Irradiation using Sole Interstitial Multicatheter Brachytherapy Versus Whole-Breast Irradiation with Boost After Breast-Conserving Surgery for Low-Risk Invasive and In-Situ Carcinoma of the Female Breast: a Randomised, Phase 3, Non-Inferiority Trial. Lancet 2016;387:229-38.

19. Polgár C, Ott OJ, Hildebrandt G, et al. Late sideeffects and cosmetic results of accelerated partial breast irradiation with interstitial brachytherapy versus wholebreast irradiation after breast-conserving surgery for lowrisk invasive and in-situ carcinoma of the female breast: 5 -year results of a randomised, controlled, phase 3 trial. Lancet Oncol 2017;18:259-68.

20. Polgár C, Major T, Fodor J, et al. Accelerated partialbreast irradiation using high-dose-rate interstitial brachytherapy: 12-year update of a prospective clinical study. Radiother Oncol 2010;94:274-9.

21. White J, Winter K, Kuske RR, et al. Long-Term Cancer Outcomes From Study NRG Oncology/RTOG 9517: A Phase 2 Study of Accelerated Partial Breast Irradiation With Multicatheter Brachytherapy After Lumpectomy for Early-Stage Breast Cancer. Int J Radiat Oncol Biol Phys 
2016;95:1460-5.

22. Rabinovitch R, Winter K, Kuske R, et al. RTOG 9517, a Phase II trial to evaluate brachytherapy as the sole method of radiation therapy for Stage I and II breast carcinoma--year-5 toxicity and cosmesis. Brachytherapy 2014;13:17-22.

23. Hattangadi JA, Powell SN, MacDonald SM, et al. Accelerated partial breast irradiation with low-dose-rate interstitial implant brachytherapy after wide local excision: 12-year outcomes from a prospective trial. Int J Radiat Oncol Biol Phys 2012;83:791-800.

24. Kamrava M, Kuske RR, Anderson B, et al. Outcomes of Breast Cancer Patients Treated with Accelerated Partial Breast Irradiation Via Multicatheter Interstitial Brachytherapy: The Pooled Registry of Multicatheter Interstitial Sites (PROMIS) Experience. Ann Surg Oncol 2015;22 Suppl 3:S404-11.

25. Benitez PR, Keisch ME, Vicini F, et al. Five-year results: the initial clinical trial of MammoSite balloon brachytherapy for partial breast irradiation in early-stage breast cancer. Am J Surg 2007;194:456-62.

26. Shah C, Badiyan S, Ben Wilkinson J, et al. Treatment efficacy with accelerated partial breast irradiation (APBI): final analysis of the American Society of Breast Surgeons MammoSite((R)) breast brachytherapy registry trial. Ann Surg Oncol 2013;20:3279-85.

27. Shah C, Khwaja S, Badiyan S, et al. Brachytherapy-based partial breast irradiation is associated with low rates of complications and excellent cosmesis. Brachytherapy 2013;12:278-84.

28. Vicini FA, Keisch M, Shah C, et al. Factors associated with optimal long-term cosmetic results in patients treated with accelerated partial breast irradiation using balloon-based brachytherapy. Int J Radiat Oncol Biol Phys 2012;83:512-8.

29. Cuttino LW, Arthur DW, Vicini F, et al. Long-term results from the Contura multilumen balloon breast brachytherapy catheter phase 4 registry trial. Int J Radiat Oncol Biol Phys 2014;90:1025-9.

30. Shah C, Wilkinson JB, Lyden M, et al. Comparison of survival and regional failure between accelerated partial breast irradiation and whole breast irradiation. Brachytherapy 2012;11:311-5.

31. Vicini FA, Remouchamps V, Wallace M, et al. Ongoing clinical experience utilizing 3D conformal external beam radiotherapy to deliver partial-breast irradiation in patients with early-stage breast cancer treated with breast-conserving therapy. Int J Radiat Oncol Biol Phys
2003;57:1247-53.

32. Formenti SC, Truong MT, Goldberg JD, et al. Prone accelerated partial breast irradiation after breastconserving surgery: preliminary clinical results and dosevolume histogram analysis. Int J Radiat Oncol Biol Phys 2004;60:493-504.

33. Formenti SC, Rosenstein B, Skinner KA, et al. T1 stage breast cancer: Adjuvant hypofractionated conformal radiation therapy to tumor bed in selected postmenopausal breast cancer patients-Pilot feasibility study. Radiology 2002;222:171-8.

34. Rodríguez N, Sanz X, Dengra J, et al. Five-year outcomes, cosmesis, and toxicity with 3-dimensional conformal external beam radiation therapy to deliver accelerated partial breast irradiation. Int J Radiat Oncol Biol Phys 2013;87:1051-7.

35. Livi L, Buonamici FB, Simontacchi G, et al. Accelerated partial breast irradiation with IMRT: new technical approach and interim analysis of acute toxicity in a phase III randomized clinical trial. Int J Radiat Oncol Biol Phys 2010;77:509-15.

36. Coles C, Agrawal R, Ah-See ML, et al. Partial breast radiotherapy for women with early breast cancer: first results of local recurrence data for IMPORT LOW (CRUK/06/003). In: European Breast Cancer Conference, 2016 Mar 9-11; Amsterdam. Eur J Cancer 2016;57. Abstract number 4LBA.

37. Coles CE, Griffin CL, Kirby AM, et al. Partial-breast radiotherapy after breast conservation surgery for patients with early breast cancer (UK IMPORT LOW trial): 5-year results from a multicentre, randomised, controlled, phase 3, non-inferiority trial. Lancet 2017;390:1048-60.

38. Meduri B, Baldissera A, Galeandro M, et al. OC-0568: Accelerated PBI VS standard radiotherapy (IRMA trial): interim cosmetic and toxicity results. Radiother Oncol 2017;123:S303.

39. De Rose F, Franceschini D, Iftode C, et al. EP-1270: Randomized phase II study of hypofractionated WBI versus APBI using VMAT: early toxicity results. Radiother Oncol 2018;127:S700.

40. Julian TB, Costantino JP, Vicini FA, et al. Early toxicity results with 3-D conformal external beam therapy (CEBT) from the NSABP B-39/RTOG 0413 accelerated partial breast irradiation (APBI) trial. Int J Radiat Oncol Biol Phys 2011;81:S7.

41. Olivotto IA, Whelan TJ, Parpia S, et al. Interim cosmetic and toxicity results from RAPID: a randomized trial of accelerated partial breast irradiation using three- 
dimensional conformal external beam radiation therapy. $\mathrm{J}$ Clin Oncol 2013;31:4038-45.

42. Belkacemi Y, Bourgier C, Kramar A, et al. SHARE: a French multicenter phase III trial comparing accelerated partial irradiation versus standard or hypofractionated whole breast irradiation in breast cancer patients at low risk of local recurrence. Clin Adv Hematol Oncol 2013;11:76-83.

43. Polgár C, Fodor J, Major T, et al. Breast-conserving treatment with partial or whole breast irradiation for low-risk invasive breast carcinoma--5-year results of a randomized trial. Int J Radiat Oncol Biol Phys 2007;69:694-702.

44. Lei RY, Leonard CE, Howell KT, et al. Four-year clinical update from a prospective trial of accelerated partial breast intensity-modulated radiotherapy (APBIMRT). Breast Cancer Res Treat 2013;140:119-33.

45. Chen PY, Wallace M, Mitchell C, et al. Four-year efficacy, cosmesis, and toxicity using three-dimensional conformal external beam radiation therapy to deliver accelerated partial breast irradiation. Int J Radiat Oncol Biol Phys 2010;76:991-7.

46. Berrang TS, Olivotto I, Kim DH, et al. Three-year outcomes of a Canadian multicenter study of accelerated partial breast irradiation using conformal radiation therapy. Int J Radiat Oncol Biol Phys 2011;81:1220-7.

47. Magee B, Swindell R, Harris M, et al. Prognostic factors for breast recurrence after conservative breast surgery and radiotherapy: results from a randomised trial. Radiother Oncol 1996;39:223-7.

48. Jagsi R, Ben-David MA, Moran JM, et al. Unacceptable cosmesis in a protocol investigating intensity-modulated radiotherapy with active breathing control for accelerated partial-breast irradiation. Int J Radiat Oncol Biol Phys 2010;76:71-8.

49. Liss AL, Ben-David MA, Jagsi R, et al. Decline of Cosmetic Outcomes Following Accelerated Partial Breast Irradiation (APBI) Using Intensity Modulated Radiation Therapy (IMRT): Results of a Single-Institution Prospective Clinical Trial. Int J Radiat Oncol Biol Phys 2013;87:S216.

50. Pashtan IM, Recht A, Ancukiewicz M, et al. External beam accelerated partial-breast irradiation using 32 gy in 8 twice-daily fractions: 5 -year results of a prospective study. Int J Radiat Oncol Biol Phys 2012;84:e271-7.

51. Hepel JT, Tokita M, MacAusland SG, et al. Toxicity of three-dimensional conformal radiotherapy for accelerated partial breast irradiation. Int J Radiat Oncol Biol Phys
2009;75:1290-6.

52. Chafe S, Moughan J, McCormick B, et al. Late toxicity and patient self-assessment of breast appearance/satisfaction on RTOG 0319: a phase 2 trial of 3-dimensional conformal radiation therapy-accelerated partial breast irradiation following lumpectomy for stages I and II breast cancer. Int J Radiat Oncol Biol Phys 2013;86:854-9.

53. Vicini F, Winter K, Wong J, et al. Initial efficacy results of RTOG 0319: three-dimensional conformal radiation therapy (3D-CRT) confined to the region of the lumpectomy cavity for stage I/ II breast carcinoma. Int J Radiat Oncol Biol Phys 2010;77:1120-7.

54. Rabinovitch R, Moughan J, Vicini F, et al. Long-term update of NRG Oncology RTOG 0319: a phase 1 and 2 trial to evaluate 3 -dimensional conformal radiation therapy confined to the region of the lumpectomy cavity for stage I and II breast carcinoma. Int J Radiat Oncol Biol Phys 2016;96:1054-9.

55. Dodwell DJ, Dyker K, Brown J, et al. A randomised study of whole-breast vs tumour-bed irradiation after local excision and axillary dissection for early breast cancer. Clin Oncol (R Coll Radiol) 2005;17:618-22.

56. Kozak KR, Smith BL, Adams J, et al. Accelerated partialbreast irradiation using proton beams: initial clinical experience. Int J Radiat Oncol Biol Phys 2006;66:691-8.

57. Livi L, Paiar F, Buonamici FB, et al. Accelerated intensitymodulated external radiotherapy as a new technical approach to treat the index quadrant after conserving surgery in early breast cancer: A preliminary study. Tumori 2005;91:227.

58. Leonard C, Carter D, Kercher J, et al. Prospective trial of accelerated partial breast intensity-modulated radiotherapy. Int J Radiat Oncol Biol Phys 2007;67:1291-8.

59. Ribeiro GG, Magee B, Swindell R, et al. The Christie Hospital breast conservation trial: an update at 8 years from inception. Clin Oncol (R Coll Radiol) 1993;5:278-83.

60. Livi L, Meattini I, Marrazzo L, et al. Accelerated partial breast irradiation using intensity-modulated radiotherapy versus whole breast irradiation: 5-year survival analysis of a phase 3 randomised controlled trial. Eur J Cancer 2015;51:451-63.

61. Peterson D, Truong PT, Parpia S, et al. Predictors of adverse cosmetic outcome in the RAPID trial: an exploratory analysis. Int J Radiat Oncol Biol Phys 2015;91:968-76.

62. Shaitelman SF, Kim LH, Grills IS, et al. Predictors of long-term toxicity using three-dimensional conformal 
external beam radiotherapy to deliver accelerated partial breast irradiation. Int J Radiat Oncol Biol Phys 2011;81:788-94.

63. Smith BD, Arthur DW, Buchholz TA, et al. Accelerated partial breast irradiation consensus statement from the American Society for Radiation Oncology (ASTRO). Int J Radiat Oncol Biol Phys 2009;74:987-1001.

64. Correa C, Harris EE, Leonardi MC, et al. Accelerated Partial Breast Irradiation: Executive summary for the update of an ASTRO Evidence-Based Consensus Statement. Pract Radiat Oncol 2017;7:73-9.

65. APBI Versus EBRT Therapy After Breast Conserving Surgery for Low-risk Breast Cancer Status: Active, not recruiting. 2018; Available online: https:// ClinicalTrials.gov/show/NCT00402519. NLM identifier: NCT00402519. Accessed September 9, 2018.

66. Standard or Hypofractionated Radiotherapy Versus Accelerated Partial Breast Irradiation (APBI)for Breast Cancer Status: Active, not recruiting. Available online: https://ClinicalTrials.gov/show/NCT01247233. NLM identifier: NCT01247233. Accessed September 9, 2018.

67. Breast Cancer With Low Risk Of Local Recurrence: Partial and Accelerated Radiation With ThreeDimensional Conformal Radiotherapy (3DCRT) Vs. Standard Radiotherapy After Conserving Surgery (Phase III Study. Available online: https://ClinicalTrials.gov/ show/NCT01803958. NLM identifier: NCT01803958. Accessed September 9, 2018.

68. Accelerated Partial Breast Irradiation With 3D-CRT and IMRT Status: Active, not recruiting. Available online: https://ClinicalTrials.gov/show/NCT02003560. NLM identifier: NCT02003560. Accessed September 9, 2018.

69. Trial of Radiation Fractionation Schedules for Once-a-Day Accelerated Partial Breast Irradiation. Available online: https://ClinicalTrials.gov/show/NCT02637024. NLM identifier: NCT02637024. Accessed September 9, 2018.

70. Accelerated Partial Breast Radiation Therapy Using HighDose Rate Brachytherapy in Treating Patients With Early Stage Breast Cancer After Surgery. Available online: https://ClinicalTrials.gov/show/NCT02526498. NLM identifier: NCT02526498. Accessed September 9, 2018.

71. 3D-CRT, Proton, or Brachytherapy APBI in Treating Patients With Invasive and Non-invasive Breast Cancer Status: Active, not recruiting. Available online: https:// ClinicalTrials.gov/show/NCT03391388. NLM identifier: NCT03391388. Accessed September 9, 2018.

72. Randomized Study on Postmenopausal Women With Early Stage Breast Cancer: WBI Versus APBI Status:
Recruiting. Available online: https://ClinicalTrials.gov/ show/NCT02375048. NLM identifier: NCT02375048. Accessed September 9, 2018.

73. APBI: $27 \mathrm{~Gy}$ in 5 Fractions for Early Breast Cancer Status: Recruiting. Available online: https://ClinicalTrials.gov/ show/NCT02681107. NLM identifier: NCT02681107. Accessed September 9, 2018.

74. APBI Proton Feasibility and Phase II Study Status: Recruiting. Available online: https://ClinicalTrials.gov/ show/NCT01839838. NLM identifier: NCT01839838. Accessed September 9, 2018.

75. Accelerated Partial Breast Irradiation for Early Breast Cancer Status: Active, not recruiting. Available online: https://ClinicalTrials.gov/show/NCT00418210. NLM identifier: NCT00418210. Accessed September 9, 2018.

76. 4D Image-Guided Partial Breast Radiation in Stage 0 and 1 Breast Cancer Status: Active, not recruiting. Available online: https://ClinicalTrials.gov/show/ NCT01646346. NLM identifier: NCT01646346. Accessed September 9, 2018.

77. Image Guided Radiotherapy (IGRT) for Prone Partial Breast Irradiation. Available online: https:// ClinicalTrials.gov/show/NCT02272400. NLM identifier: NCT02272400. Accessed September 9, 2018.

78. BrUOG 291: Five Fraction Partial Breast Irradiation Using Non-invasive Image-guided Breast Brachytherapy (NIBB). Available online: https://ClinicalTrials.gov/ show/NCT01961531. NLM identifier: NCT01961531. Accessed September 9, 2018.

79. A Multi-Institutional Registry for CyberKnife Stereotactic Accelerated Partial Breast Irradiation (CK-SAPBI) Status: Recruiting. Available online: https://ClinicalTrials.gov/ show/NCT02457117. NLM identifier: NCT02457117. Accessed September 9, 2018.

80. Radiation Therapy (WBI Versus PBI) in Treating Women Who Have Undergone Surgery For Ductal Carcinoma In Situ or Stage I or Stage II Breast Cancer Status: Active, not recruiting. Available online: https://ClinicalTrials.gov/ show/NCT00103181. NLM identifier: NCT00103181. Accessed September 9, 2018.

81. Assessing the Cosmesis and Toxicity of Partial Breast Irradiation Using Proton Beam Irradiation. Available online: https://ClinicalTrials.gov/show/ NCT01245712. NLM identifier: NCT01245712. Accessed September 9, 2018.

82. Comparing Sequential Neoadjuvant Treatment Including Chemotherapy and Accelerated Radiation Focused to the Tumor Bed vs Neoadjuvant Chemotherapy Alone. 
Available online: https://ClinicalTrials.gov/show/ NCT02806258. NLM identifier: NCT02806258. Accessed September 9, 2018.

83. Preoperative Accelerated Partial Breast Irradiation for Triple Negative Breast Cancer Using Proton Beam Scanning. Available online: https://ClinicalTrials.gov/ show/NCT03340402. NLM identifier: NCT03340402.
Accessed September 9, 2018.

84. Preoperative Accelerated Partial Breast Irradiation (APBI) for Women With Stage I and Select IIA Breast Cancer. Available online: https://ClinicalTrials.gov/ show/NCT01014715. NLM identifier: NCT01014715. Accessed September 9, 2018.
Cite this article as: Bennion NR, Baine M, Granatowicz A, Wahl AO. Accelerated partial breast radiotherapy: a review of the literature and future directions. Gland Surg 2018;7(6):596-610. doi: $10.21037 /$ gs.2018.11.05 